\title{
Performance of Tightly Coupled Integration of GPS/BDS/MEMS- INS/Odometer for Real-Time High-Precision Vehicle Positioning in Urban Degraded and Denied Environment
}

\author{
Fei Liu $\mathbb{D}^{1},{ }^{1}$ Houzeng Han $\mathbb{D},{ }^{2}$ Xin Cheng, ${ }^{2}$ and Binghao Li $\mathbb{C}^{3}$ \\ ${ }^{1}$ School of Environment Science and Spatial Informatics, China University of Mining and Technology (CUMT), \\ Xuzhou 221116, China \\ ${ }^{2}$ School of Geomatics and Urban Spatial Informatics, Beijing University of Civil Engineering and Architecture (BUCEA), \\ Beijing 102616, China \\ ${ }^{3}$ School of Minerals and Energy Resources Engineering, University of New South Wales (UNSW), Sydney 2053, Australia \\ Correspondence should be addressed to Houzeng Han; hanhouzeng@bucea.edu.cn
}

Received 29 September 2019; Revised 18 November 2019; Accepted 4 December 2019; Published 28 February 2020

Academic Editor: Valerie Renaudin

Copyright $\odot 2020$ Fei Liu et al. This is an open access article distributed under the Creative Commons Attribution License, which permits unrestricted use, distribution, and reproduction in any medium, provided the original work is properly cited.

\begin{abstract}
Global Navigation Satellite System Real-Time Kinematic (GNSS-RTK) technology is widely used in vehicle navigation, but in complex environments such as urban high-rise street, wooded street, overpass, and tunnel, satellite signals are prone to attenuation or even unavailability. It brings great challenges to the continuous high-precision navigation. For this reason, a tightly coupled (TC) integration algorithm for GPS (Global Positioning System)/BDS (BeiDou Navigation Satellite System)/MEMS-INS (Micro-Electro-Mechanical System-Inertial Navigation System)/Odometer (GCIO) is proposed for vehicle navigation in complex urban environments. The accuracy improvement and ambiguity resolution (AR) performance are analysed in this research. First of all, the INS positioning error is constrained by fusion GPS/BDS (GC) and odometer; then, the predicted position information is used to aid GPS/BDS ambiguity resolution. In GNSS-denied environments, the odometer/INS integration is still carried out for continuous navigation. Real-time experiments are carried out in urban degraded and denied environments to validate the performance of the integrated system. In high-rise streets, the ambiguity fixing success rate of GCIO mode is $13.57 \%$ higher than that of GC mode. In the wooded street environment, the success rate has increased particularly significantly, by about 55 percent. The positioning accuracy analysis for open environment, high-rise street, wooded street, overpass, and tunnel is conducted. The experimental results show that in the above environment, the order of $0.1 \mathrm{~m}$ positioning accuracy can be achieved in the case of satellite outage for 1 minute, which can meet the positioning needs in most scenarios.
\end{abstract}

\section{Introduction}

High-precision navigation and position information are the key components of autopilot technology $[1,2]$. In the outdoor open environment, GPS/BDS/GLONASS $[3,4]$ (global navigation satellite system) and other constellation systems can provide far superior satellite geometry and signal availability and more consistent and accurate positioning capability, which can basically meet the needs of high-precision position information of vehicles [5-7]. However, the number of visible/available satellites in high-rise buildings, three- dimensional traffic, long-distance tunnels, underground parking, and other complex urban environments is decreasing, even the satellite signal is not available for positioning, which brings great challenges to vehicle navigation [8-10]. GNSS/INS integrated system can provide better performance than simple GNSS in complex environments $[9,11,12]$; however, the performance of GNSS/INS integration depends heavily on the quality of Inertial Measurement Unit (IMU) adopted. The positioning accuracy will degrade rapidly while there is GNSS interruption and blockage if only low-cost INS is applied $[13,14]$. 


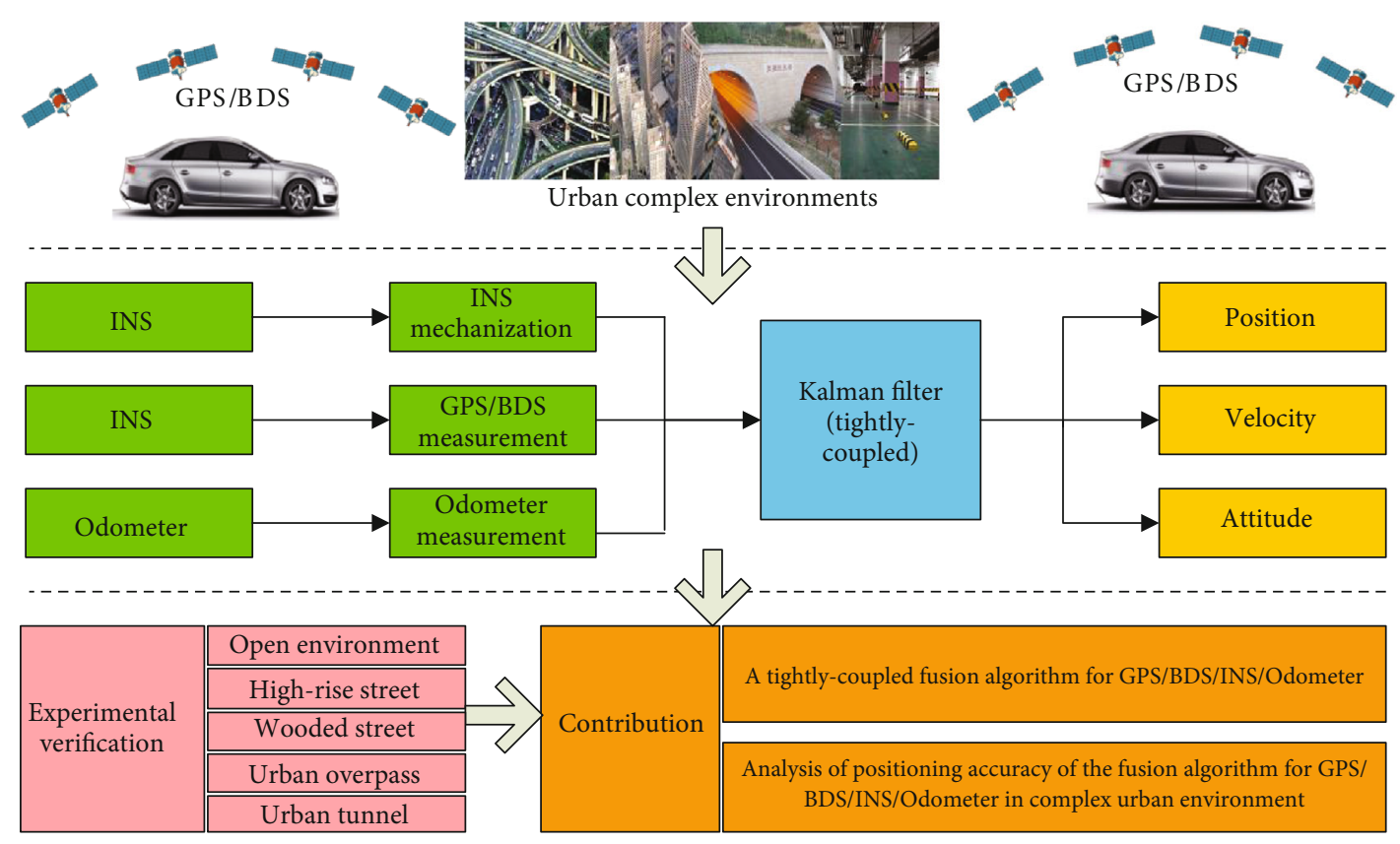

FIgURE 1: Technology roadmap.

In order to obtain better positioning performance of the vehicles, it needs some assistant technologies to improve the integrity and accuracy of GNSS/INS navigation system, such as an odometer $[15,16]$. There are a few reports in the literature on combining GNSS/INS/Odometer. Li et al. [16] propose a fuzzy neural network (FNN) for land vehicle navigation applications based on GNSS/INS/Odometer, which can improve the position, velocity, and attitude accuracy of the integrated system, especially the position parameters, over long GPS outages. However, the positioning accuracy and accuracy stability of urban interchange, tunnel, and other complex conditions have not been analysed and explained. Park et al. [17] put forward an integrated navigation system based on GNSS/INS/odometer/barometer for vehicle navigation; when GNSS signals are not available for 20 seconds, the plane positioning accuracy is $23.85 \mathrm{~m}$, and the elevation positioning accuracy is $1.28 \mathrm{~m}$. It can be seen that the positioning accuracy is relatively low. Aftatah et al. [18] propose a loosely coupled solution that fuses the GPS/IN$\mathrm{S} /$ Odometer data. In the three zones which have duration of 70 seconds of GPS outages in total, the positioning accuracy of the order of $0.3 \mathrm{~m}$ is realized. However, this situation can only show the behaviour accuracy of GPS signal when it is disturbed for a short time. In addition, other scholars [19-21] have carried out the researches of GNSS/INS/Odometer integrated navigation technology; the results show that in the case of GNSS unlocked, it can still maintain certain accuracy in a short time. However, the relevant papers still have some shortcomings and insufficient in analysing and explaining the positioning accuracy in the common scenes of wooded streets, tunnels, and other urban complicated environments.
This paper focuses on the problem of high-precision vehicle positioning in complex urban environment and carries out the research of tight combination algorithm based on GCIO. The positioning-related indexes of the fusion of GC and the positioning accuracy of GCIO in the urban environment such as open environment, highrise street, wooded street, overpass, and tunnel are analysed. Finally, the main contributions of this paper are summarized, including a tightly coupled fusion algorithm for GCIO and the analysis of positioning accuracy of a tightly coupled fusion algorithm in complex urban environment. The technology roadmap is shown in Figure 1.

The remainder of this paper is organized as follows. Section 2 briefly reviews the tightly coupled GCIO integration model, including the dynamic model and measurement model. The experiment of multisensor fusion positioning is carried out in Section 3, and the positioning accuracy based on multisensor fusion in the complex urban environment is discussed and analysed. In Section 4, some conclusions related to the work are summarized.

\section{Methodology}

An overview of the TC integration of GCIO algorithm is shown in Figure 2 [22]. After initialization of the tightly coupled algorithm, the compensated raw INS data is used to provide the continuous navigation result (position, velocity, and attitude) through INS mechanization. The velocity information of odometer is fused with INS to correct the integral error of INS and reduce the error accumulation. When GNSS is available, the double-difference (DD) pseudorange and carrier phase observations from rover receiver and base receiver are used for KF (Kalman filter) 


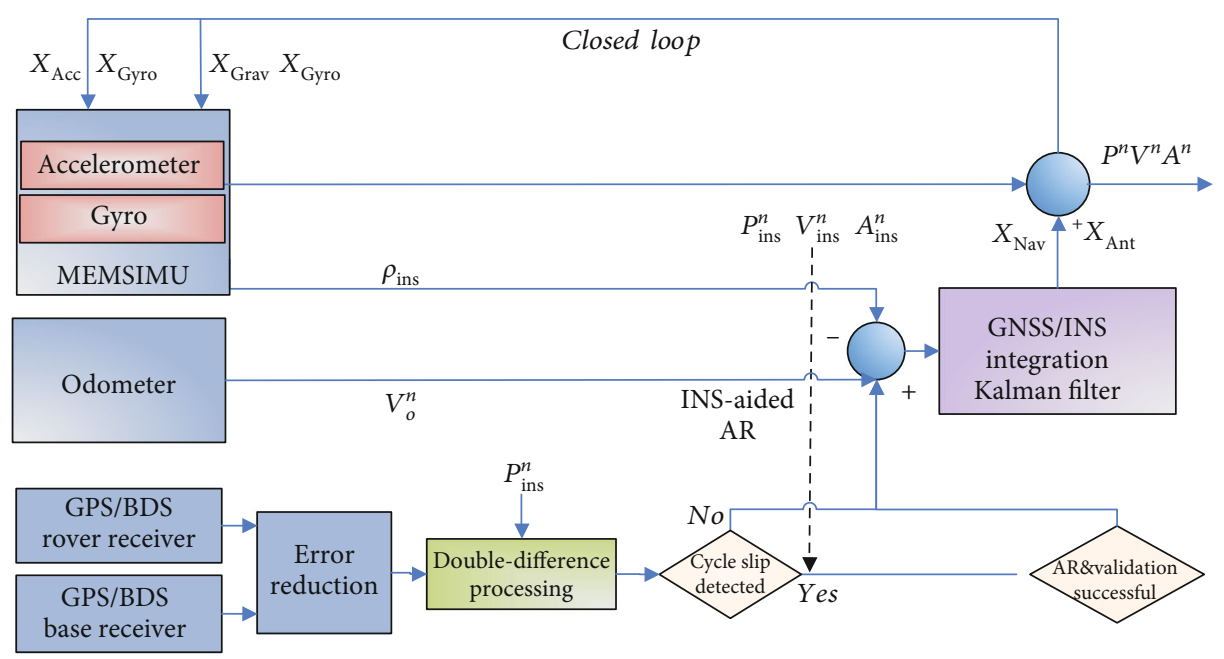

FIgURE 2: Algorithm frame of the TC GPS/BDS/INS/Odometer integration.

measurement update. Then, GCIO data are fused in the tightly coupled model to obtain high-accuracy position, velocity, and attitude information. When GNSS outage occurs, INS/Odometer integration is used to obtain continuous navigation results.

2.1. GPS/BDS RTK Model. The DD technique can remove the error sources that are highly correlated over space and time, such as ephemeris errors [23]. Range information with remained multipath and receiver noise can achieve cm-level accuracy only after the integer ambiguities in the carrier phase measurements have been resolved correctly [24]. In a general form, the DD code and carrier phase observation model can be described as $[22,25,26]$

$$
\left\{\begin{array}{l}
\Delta \nabla \rho=\Delta \nabla \rho_{0}+\Delta \nabla T+\Delta \nabla I+\Delta \nabla \mathrm{M}+\Delta \nabla \varepsilon_{\rho}, \\
\lambda \Delta \nabla \phi=\Delta \nabla \phi_{0}+\Delta \nabla T-\Delta \nabla I+\lambda \Delta \nabla N+\Delta \nabla m+\Delta \nabla \varepsilon_{\phi},
\end{array}\right.
$$

where the $\Delta \nabla$ notation refers to a double-differentiation. $\rho$ and $\phi$ denote the pseudorange and carrier phase observations, the geometric distance between the receiver and satellite is $\rho_{0}, T$ is tropospheric delay, $I$ is ionospheric delay, $\lambda$ represents the carrier phase wavelength, and $M$ is the pseudorange multipath error and $m$ denotes the carrier phase multipath error; $\varepsilon_{\rho}$ and $\varepsilon_{\phi}$ are the pseudorange noise and carrier phase noise.

For GNSS-RTK with GPS and BDS data, the equation to estimate the unknown parameters can then be written as follows:

$$
\left[\begin{array}{lll}
H^{G} & \Lambda^{G} & \\
H^{C} & & \Lambda^{C}
\end{array}\right]\left[\begin{array}{c}
\delta p_{r} \\
\nabla \Delta N^{G} \\
\nabla \Delta N^{C}
\end{array}\right]=\left[\begin{array}{c}
\nabla \Delta L^{G} \\
\nabla \Delta L^{C}
\end{array}\right],
$$

where the superscripts " $G$ " and " $C$ " represent GPS and BDS, respectively; $H$ is the geometry matrix containing satellitegeometry information; $\partial p_{r}$ denotes the vector of baseline increment; $\Lambda$ is the coefficient matrix of ambiguities; $\nabla \Delta L$ is the DD observed minus computed vector.

With the increase of the distance between the vehicle and the base station, the baseline length increases gradually, and the atmospheric residual error in formulas (1) and (2) cannot be eliminated by DGNSS. As a result, the dynamic relative positioning accuracy and reliability are not as good as the short baseline scenario. For the dynamic positioning accuracy of the medium/long baseline, the method of [27] is adopted.

In order to characterize the probability of correct fixing, the ambiguity success rate has been introduced to evaluate the strength of the underlying model, where the bootstrapped success rate is often used as lower bounding for integer least-square success rate [28]:

$$
P_{s} \geq \prod_{i=1}^{n}\left[2 \Phi\left(\frac{1}{2 \sigma_{\bar{z}_{i||}}}\right)-1\right], \Phi(x)=\frac{1}{\sqrt{2 \pi}} \int_{-\infty}^{x} e^{-(1 / 2) t^{2}} d t
$$

where $z$ is the true ambiguity vector, and $\sigma_{\widehat{z}}$ denotes the standard deviation of the conditional ambiguities.

2.2. Odometer Navigation. An odometer is a device that can independently measure the distance and speed of the vehicle, which can measure the speed of the vehicle in the forward direction $[16,29]$. Assuming that the vehicle velocity of the odometer output is $v_{O}$, which points directly in front of the vehicle, that is, parallel to the $Y$ axis of the vehicle coordinate system, the three-dimensional velocity in the vehicle coordinate system is expressed in vector form, so the expression of the vehicle velocity in the navigation coordinate system is

$$
v_{O}^{n}=C_{b}^{n}\left[\begin{array}{lll}
0 & v_{O} & 0
\end{array}\right]^{T} .
$$


Among them, $C_{b}^{n}$ is the direction cosine matrix from the vehicle coordinate system to the navigation coordinate system that can be calculated by the inertial navigation system.

The integral of the velocity can get the position information in the navigation coordinate system, and the difference equation of the position can be written as follows:

$$
\begin{aligned}
& \dot{L}_{O}=\frac{v_{O N}^{n}}{R_{M}+h_{O}}, \\
& \dot{\lambda}_{O}=\frac{v_{O E}^{n}}{\left(R_{N}+h_{O}\right) \cos L_{O}}, \\
& \dot{h}_{O}=-v_{O D}^{n} .
\end{aligned}
$$

Among them, $L_{O}, \lambda_{O}$, and $h_{O}$ are the longitude, latitude, and height, respectively. $R_{M}$ and $R_{N}$ are the curvature radius of the meridian circle and prime vertical of the earth, where the location of vehicle, respectively, and $v_{O N}^{n}, v_{O E}^{n}$, and $v_{O D}^{n}$ are the velocity components in the north, east, and earth directions in the navigation coordinate system, respectively.

The velocity information acquisition of odometer belongs to discrete sampling, and the position calculation method of odometer can be deduced based on the difference equation.

$$
\begin{aligned}
& L_{O}(k)=L_{O}(k-1)+\frac{v_{O N}^{n} \bullet \Delta t}{R_{M}+h}, \\
& \lambda_{O}(k)=\lambda_{O}(k-1)+\frac{v_{O E}^{n} \bullet \Delta t}{\left(R_{N}+h\right) \cos L_{O}(k-1)}, \\
& h_{O}(k)=h_{O}(k-1)-v_{O D}^{n} \bullet \Delta t .
\end{aligned}
$$

Among them, $(k-1)$ indicates the odometer information at $(k-1)$ time, $(k)$ represents the odometer information at $k$ time, and $\Delta t$ is the odometer sampling time interval.

Since the odometer has no zero deviation, the speed model of the odometer output can be expressed as

$$
\tilde{V}_{O}=[1+\delta k]+V_{O}+w_{O} .
$$

In the formula, $\delta k$ is the error of calibration coefficient, and $w_{O}$ is the observation.

2.3. AR Strategy Based on INS. High-precision carrier phase positioning technology depends on high reliability and accurate integer ambiguity resolution. However, in the urban degraded and denied environment, especially in the moving state, the GNSS often appears the outage state, which leads to the ambiguity resolution error. In
TABLE 1: Main performance specifications of the INS sensors.

\begin{tabular}{lcc}
\hline Parameter & MEMS INS & FOG INS \\
\hline Accelerometer bias stability $(\mathrm{mg})$ & 2 & 0.2 \\
Velocity random walk $(\mu \mathrm{g} / \mathrm{sqrt}(\mathrm{Hz}))$ & 120 & 20 \\
Gyro bias stability $(\mathrm{deg} / \mathrm{h})$ & 5 & 0.05 \\
Angular random walk $(\mathrm{deg} / \mathrm{sqrt}(\mathrm{h}))$ & 0.4 & 0.005 \\
\hline
\end{tabular}

GPS/BDS/INS combination positioning, because INS can provide continuous positioning ability, the accuracy of ambiguity floating point solution is improved, and then, the ambiguity search space is constrained and the ambiguity solution efficiency is improved. The ambiguity solution equation of combined system is obtained [22,30].

$$
\underbrace{\left[\begin{array}{c}
\lambda \Delta \nabla \phi-\Delta \nabla \rho_{0} \\
\Delta \nabla \rho-\Delta \nabla \rho_{0} \\
0_{3 \times 1}
\end{array}\right]}_{L}=\underbrace{\left[\begin{array}{cc}
H_{b} & \lambda I_{n \times n} \\
H_{b} & 0_{n \times n} \\
I_{3 \times 3} & 0_{n \times n}
\end{array}\right]}_{H} \underbrace{\left[\begin{array}{c}
X_{b} \\
X_{a}
\end{array}\right]}_{X}+\underbrace{\left[\begin{array}{c}
e_{\phi} \\
e_{\rho} \\
e_{\text {ins }}
\end{array}\right]}_{e},
$$

where $X_{b}$ is the correction number of position error, $X_{a}$ is the parameter vector of $n$-dimensional ambiguity, $H_{b}$ is the design matrix, and $e_{\text {ins }}$ is the observation noise of INS system, which can be obtained from a priori equation matrix of combined filtering.

\subsection{Tightly Coupled Integration of GPS/BDS/MEMS- INS/Odometer}

2.4.1. INS Dynamic Model. In this research, a tightly coupled integration algorithm of GPS/BDS/INS/Odometer is adopted with the EKF (extended Kalman filter); the inertial dynamic model is as follows [31]:

$$
\begin{aligned}
\delta \dot{v} & =-\left(2 \omega_{i e}+\omega_{e n}\right) \times \delta v-\delta \psi \times f+\nabla, \\
\delta \dot{r} & =-\omega_{e n} \times \delta r+\delta v, \\
\delta \dot{\psi} & =-\left(\omega_{i e}+\omega_{e n}\right) \times \delta \psi+\varepsilon,
\end{aligned}
$$

where $\delta r, \delta v$, and $\delta \psi$ are the error vectors of position, velocity, and attitude, respectively, $\nabla$ and $\varepsilon$ are the error vectors of accelerometer and gyro, respectively, $f$ is the specific force vector, $\omega_{i e}$ is the earth rotation vector, and $\omega_{e n}$ is the craft rate.

An INS error model with twenty-seven states is implemented here, which contains nine navigation error states that are expressed in the north-east-down (NED) navigation frame (three for position, three for velocity, and three for orientation), six accelerometer and six 

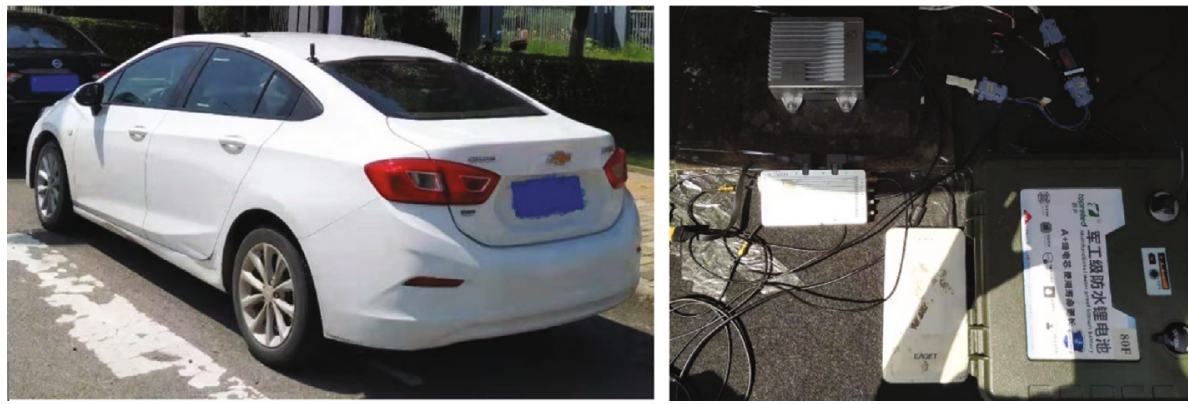

FIgURE 3: Schematic diagram of installation of experimental vehicles and equipment.

TABLE 2: Main performance specifications of the FOG GNSS/INS combination system.

\begin{tabular}{lc}
\hline Parameter & GNSS/INS \\
\hline Positioning accuracy in RTK model & $2 \mathrm{~cm}+1 \mathrm{ppm}$ \\
Attitude measurement accuracy (deg) & 0.01 \\
Heading measurement accuracy (deg) & 0.02 \\
\hline
\end{tabular}

gyro sensor errors (including three biases and three scale factors for each axis), three gravity uncertainty errors, and three lever arm errors [22, 32]. The detailed error states are given as follows:

$$
\left\{\begin{array}{l}
X_{\mathrm{NAV}}=\left[\delta r_{N}, \delta r_{E}, \delta r_{D}, \delta v_{N}, \delta v_{E}, \delta v_{D}, \delta \psi_{N}, \delta \psi_{E}, \delta \psi_{D}\right]^{T}, \\
X_{\mathrm{Acc}}=\left[\nabla_{b x}, \nabla_{b y}, \nabla_{b z}, \nabla_{f x}, \nabla_{f y}, \nabla_{f z}\right]^{T}, \\
X_{\mathrm{Gyro}}=\left[\varepsilon_{b x}, \varepsilon_{b y}, \varepsilon_{b z}, \varepsilon_{f x}, \varepsilon_{f y}, \varepsilon_{f z}\right]^{T}, \\
X_{\text {Ant }}=\left[\delta L_{b x}, \delta L_{b y}, \delta L_{b z}\right]^{T} .
\end{array}\right.
$$

The inertial sensor errors are modelled as the firstorder Gauss-Markov (GM) processes, while the float ambiguities are treated as constant over time. Additional atmospheric error states are added for long-baseline configuration.

2.4.2. Multisensor Fusion Model. A TC EKF system model considering psi-angle error, INS sensor error, and odometer scale factor is designed as follows:

$$
\dot{X}_{\mathrm{INS}}=F X_{\mathrm{INS}}+G \omega
$$

where $F$ is the dynamic matrix, and $G$ is the noise distribution matrix. The state vector $X$ can be written as

$$
\mathrm{X}=\left[X_{\mathrm{Nav}}, X_{\mathrm{Acc}}, X_{\mathrm{Gyro}}, X_{\mathrm{Ant}}, \delta k\right]^{T} \text {, }
$$

where $\delta k$ is the error of calibration coefficient.

When the GPS/BDS, INS, and odometer observable data arrive at the same epoch, the tightly coupled GPS/BDS/INS/Odometer measurement model will be used to update the EKF. The measurement model for EKF in the discrete time domain is expressed as

$$
Z_{k}=H_{k} X_{k}+\eta_{k}
$$

where $H_{k}$ is the design matrix, and $Z_{k}$ is the measurement vector whose value is the differences between INSpredicted measurement, GNSS raw observables, and odometer data:

$$
\begin{aligned}
& \mathrm{Z}_{k}=\left[\begin{array}{c}
\nabla \Delta \widehat{\rho}_{\mathrm{INS}}-\nabla \Delta P_{\mathrm{GPS} \mid \mathrm{BDS}} \\
\nabla \Delta \widehat{\rho}_{\mathrm{INS}}-\lambda \nabla \Delta \varphi_{\mathrm{GPS} \mid \mathrm{BDS}} \\
V_{\mathrm{INS}}-V_{\mathrm{Odo}}
\end{array}\right] \\
& {\left[V_{\mathrm{INS}}-V_{O}\right]=\left[\begin{array}{c}
\left(V_{\mathrm{INS}}^{n}-V_{o}^{n}\right)_{N} \\
\left(V_{\mathrm{INS}}^{n}-V_{o}^{n}\right)_{E} \\
\left(V_{\mathrm{INS}}^{n}-V_{o}^{n}\right)_{D}
\end{array}\right]} \\
& =\left[\begin{array}{cccc}
1 & 0 & 0 & R_{o 12}^{n} V_{O} \\
0 & 1 & 0 & R_{o 22}^{n} V_{O} \\
0 & 0 & 1 & R_{o 32}^{n} V_{O}
\end{array}\right]\left[\begin{array}{c}
\delta V_{N} \\
\delta V_{E} \\
\delta V_{D} \\
\delta k
\end{array}\right] \text {, }
\end{aligned}
$$

where the subscripts "INS" and "GPS|BDS" represent the predicted GPS|BDS measurements using INS-derived information and observables from the GPS|BDS receivers, respectively. $V_{\text {INS }}^{n}$ and $V_{o}^{n}$ represent the velocity values calculated in the navigation coordinate system by INS and odometer, respectively. NED represents the NE geodetic coordinate system. The linearized form of INS-predicted DD observation can be written as

$$
\nabla \Delta \widehat{\rho}+\left(\overrightarrow{e_{k}}-\overrightarrow{e_{j}}\right) \cdot \delta r^{e}
$$

where $\overrightarrow{e_{k}}$ and $\overrightarrow{e_{j}}$ are the unit line-of-sight (LOS) vectors from the INS center to the $k$ th and $j$ th satellite, respectively; $\delta r^{e}$ is the position error vector expressed in the $e$-frame. 

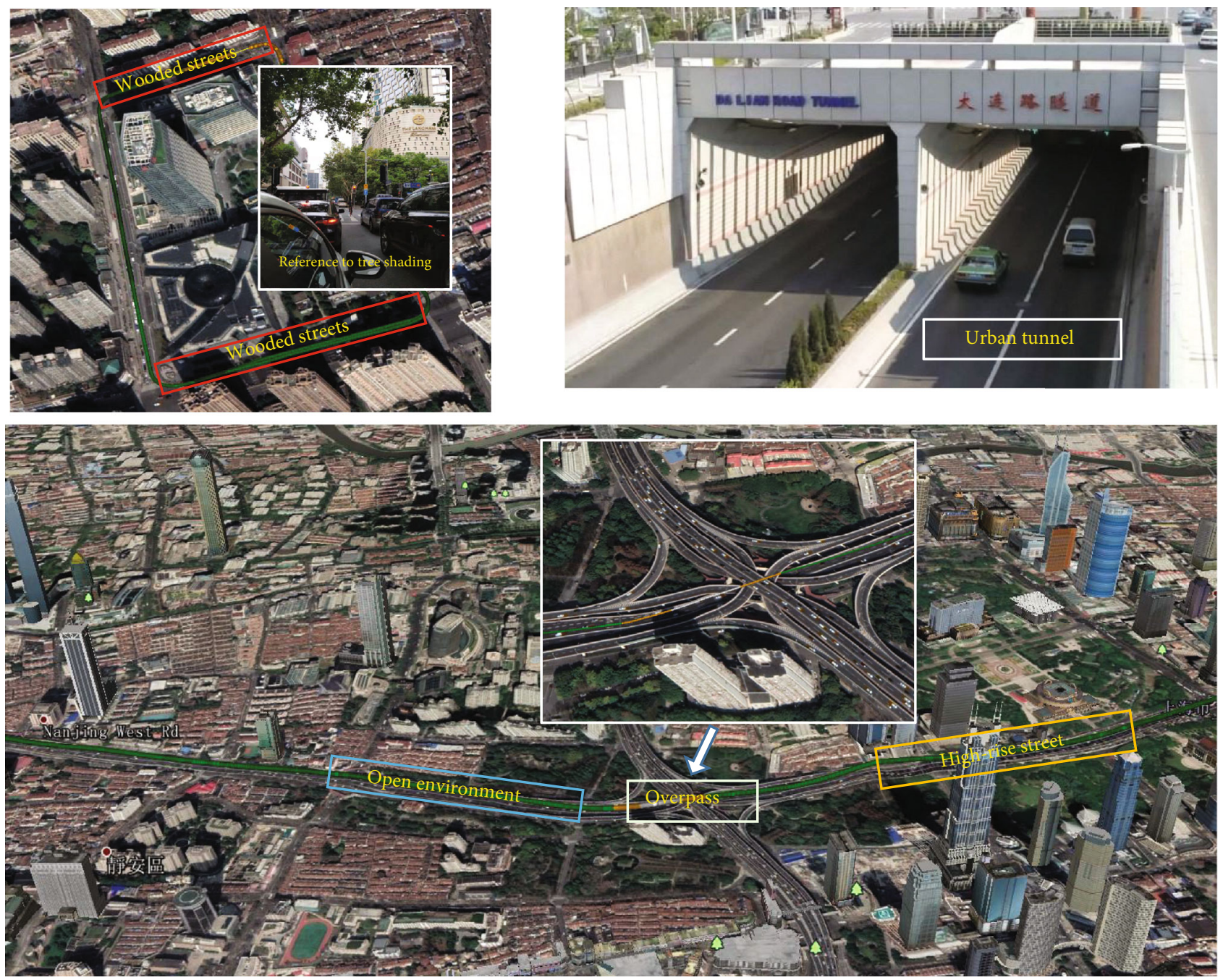

FIgURE 4: Actual test route and vehicle trajectory and schematic diagram of 5 scenarios.

\section{Experiment and Discussion}

In order to verify the technical method of this paper, the vehicle experiment was carried out in Shanghai. The experimental equipment is a tightly combined GNSS/INS module developed by ourselves and is connected to the odometer of the vehicle through the cable. The performance metrics of INS devices are shown in Table 1. The equipment is installed in the trunk of the vehicle, the GPS/BDS antenna is installed on top of the vehicle, and the experimental data are transmitted to the notebook computer via cable. RTK is supported by domestic GNSS core stations. The system integration is shown in Figure 3.

In order to verify the positioning accuracy, a highprecision fiber-optic gyroscope (FOG) GNSS/INS combination system produced by No. 707 Research Institute of China Shipbuilding Industry Corporation is used as a precise comparison reference. The parameters of the MEMS INS, FOG INS, and GNSS/FOG INS are shown in Tables 1 and 2 .
The experiment is aimed at 5 scenarios: urban open environment, wooded streets, urban tunnel, overpass, and high-rise street. The specific test route is shown in Figure 4 . The speed of the vehicle during the test is shown in Figure 5.

In the course of the experiment, the vehicle speed is controlled at $10-80 \mathrm{~km} / \mathrm{h}$, which basically covers the driving speed in the urban environment. The details are shown in Figure 5.

As can be seen from Figures 4 and 5, this test has experienced the most urban driving environments and basically simulated the vehicle speed in the normal driving process.

3.1. Analysis of Satellite Visibility and Dilution of Precision (DOP) Value. Overpasses, high-rise and tree-sheltered streets, and other urban environments would have an impact on the vehicle satellite receiver in terms of satellite cut-off angle and signal reception, which leads to a decrease in the number of visible satellites and DOP values. To illustrate the availability of GPS/BDS in urban environments, the number and DOP values of GPS/BDS satellites in 6 


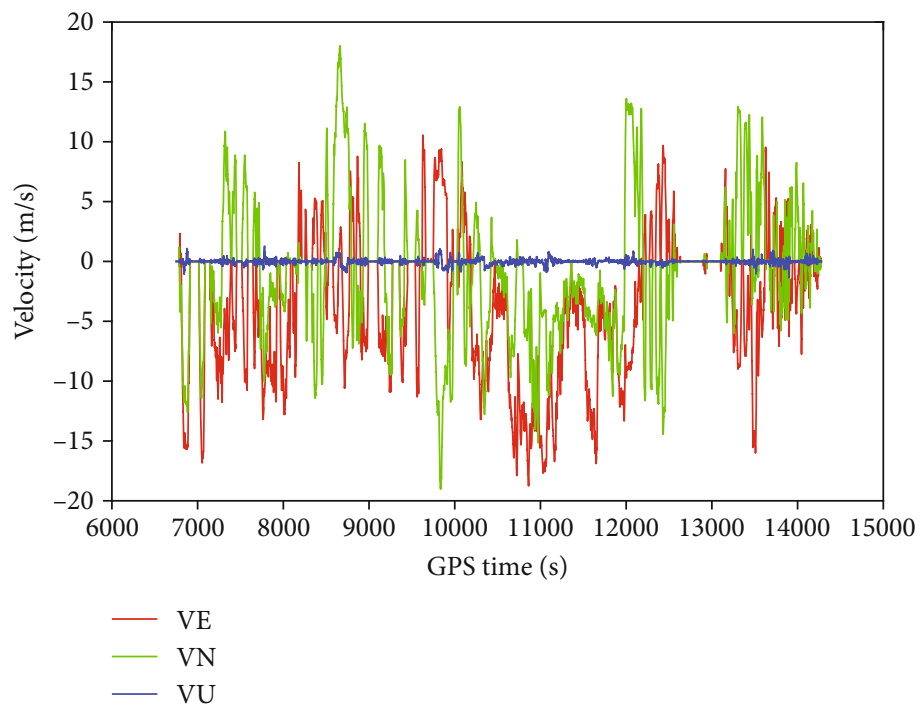

FIgURe 5: The speed of the vehicle during the test.

cut-off angles from $10^{\circ}$ to $35^{\circ}$ as well as the above three real scenes are simulated and analysed. In addition, for the long-distance tunnel and other environments, because the satellite is in the state of outage, the related performance is no longer analysed.

As can be seen from Figure 6, in an open urban environment, such as an open ring road, the number of the satellites can basically be maintained at 20 at $10^{\circ}$ and $15^{\circ}$ cut-off angle, which can meet the navigation needs of the number of satellites and geometric structure. With the increase of the cut-off angle, the satellite data gradually decreased to 14 at $35^{\circ}$ cut-off angle. Within the range, the number and geometry of available satellites will be affected, and as can be seen from Table 3, the PDOP (Position Dilution of Precision) value at this time is about $63.89 \%$ lower than that at $15^{\circ}$ cut-off angle. In addition, the accuracy of VDOP is almost more than 2-3 times worse than that of HDOP (Horizontal Dilution of Precision). It can be seen that the height cut-off angle has a greater influence on the VDOP (Vertical Dilution of Precision) of the satellite. With the increase of the height cut-off angle, the DOP value decreases gradually, which also shows from the side that a large number of high-rise streets, tree-sheltered streets, and overpasses will have an impact on the number and geometry of satellites.

Figure 7 shows the number and DOPs of GPS/BDS satellites in high-rise streets, wooded streets, and overpass. Compared with the open environment in Figure 6, the number of satellites decreases and fluctuates and varies greatly from scene to scene. Compared with the three scenarios, in the high-rise street, the number of satellites fluctuates between 10 and 20; in the wooded street, the number of satellites fluctuates between 5 and 15, and the total number is lower than that in the high-rise street environment. In the urban overpass environment, the number of satellites will decline rapidly until it falls to 0 .

Table 4 shows the satellite DOP values in the above environments. Because the number of satellites under the over-

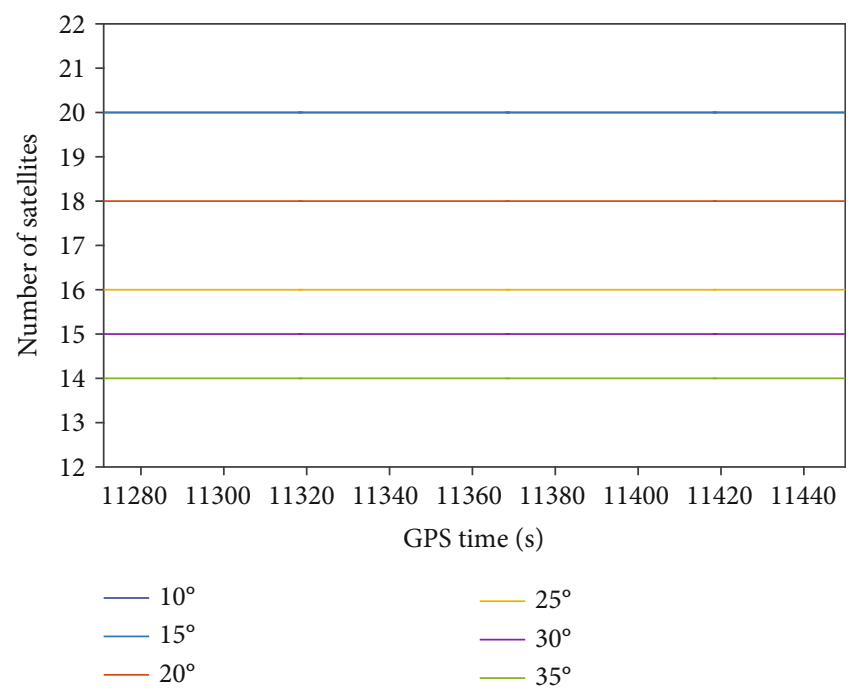

FIgURE 6: The number of GPS/BDS satellites in urban open environment.

TABLe 3: Mean DOPs of GPS+BDS for $10^{\circ}$ to $35^{\circ}$ cut-off elevation angles.

\begin{tabular}{lcccccc}
\hline Cut-off angle $\left(^{\circ}\right)$ & 10 & 15 & 20 & 25 & 30 & 35 \\
\hline PDOP & 1.3000 & 1.3000 & 1.8000 & 2.0356 & 2.5222 & 3.6000 \\
HDOP & 0.6000 & 0.6000 & 0.8000 & 0.8000 & 0.9000 & 1.1000 \\
VDOP & 1.1000 & 1.1000 & 1.6072 & 1.8761 & 2.3750 & 3.4000 \\
\hline
\end{tabular}

pass is zero, DOP cannot be calculated. Based on the analysis of Figure 7 and Table 4, it can be seen that all the above environments have an impact on the positioning performance of satellites. As can be seen from Tables 3 and 4, DOP is relatively good in the high-rise street environment but still worse than in the open environment. However, the 

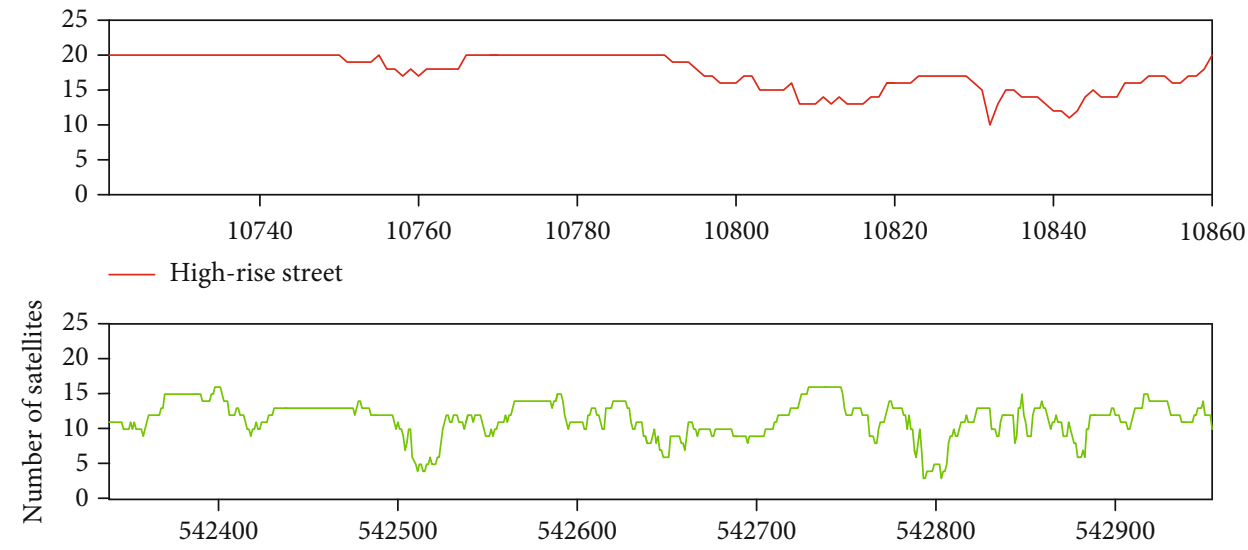

Wooded street

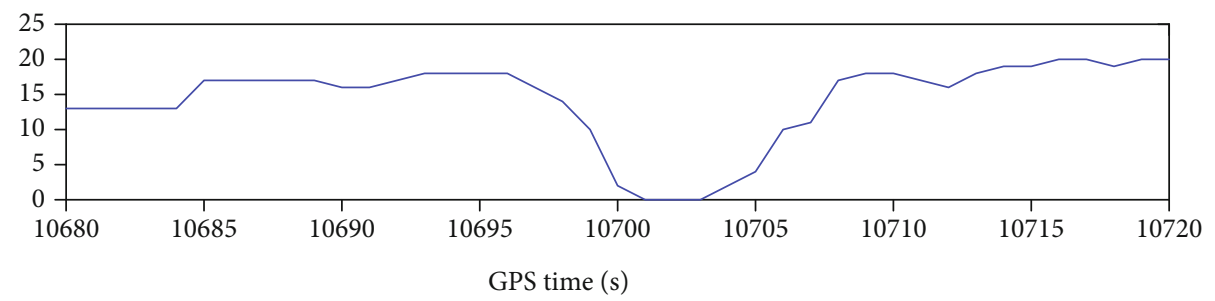

- Overpass

FIGURE 7: The number of GPS/BDS satellites in high-rise streets, wooded streets, and overpass.

TABle 4: Mean DOPs of GPS/BDS in high-rise street, wooded street, and overpass at $15^{\circ}$ cut-off angle.

\begin{tabular}{lccc}
\hline Scene & High-rise street & Wooded street & Overpass \\
\hline PDOP & 1.6343 & 5.6259 & - \\
HDOP & 0.7300 & 1.8928 & - \\
VDOP & 1.4379 & 5.2026 & - \\
\hline
\end{tabular}

DOP value under wooded street is relatively poor, even lower than the DOP value when the cut-off angle is $30^{\circ}$, indicating that this environment has a great influence on the navigation accuracy in the city. Analogy can be seen that the positioning accuracy of the road under the urban viaduct will also be relatively poor. Under the complex overpass, there will even be the unlocked state of satellite positioning.

Generally speaking, in the urban environment, only relying on satellite positioning system can meet the navigation needs of more environments. However, even if the environment such as underground garages is not considered, the positioning accuracy and reliability will be greatly affected in the above-mentioned urban satellite navigation signal-degraded and signal-denied environment. For this reason, it is necessary to introduce other sensors to support the positioning requirements when the satellite is outage.

3.2. Analysis of AR Performance. The correct resolution of integer ambiguity is an important link to achieve highprecision positioning, but in degraded and denied environ- ment, the satellite signal is easy to lose, resulting in inaccurate ambiguity resolution. In this paper, the AR performance in terms of solution state from the AR process and the empirical success rate would be compared and analysed in urban open environment and complex environment. The solution state from the AR process includes " 0 " and " 1 ," in which " 0 " indicates failure to fix ambiguity and " 1 " indicates success in fixing ambiguity. Details are shown in Figure 8 and Table 5.

The solution state can be used to indicate the probability of correctly fixing the integer ambiguities. Figure 8 shows the solution state of GPS/BDS and GPS/BDS/IN$\mathrm{S} /$ Odometer combinations in high-rise street and wooded street. In most cases of GPS/BDS, the ambiguity is fixed, while in the case of GPS/BDS/INS/Odometer combination, the ambiguity is all fixed. In the wooded street, the GPS/BDS ambiguity is much worse than that in the high-rise street, but in the case of GPS/BDS/INS/Odometer combination, only a few of the ambiguities are not fixed. It can be seen that the GPS/BDS/INS/Odometer combination is of great help to the fixation of ambiguity, especially in wooded streets.

The empirical success rate of AR for different scenes of open environment, high-rise streets, wooded streets, and overpass is also investigated. The resolved ambiguities will be compared with the reference ambiguities to ensure the correctness of the fixed ambiguities. The empirical success rate can be defined as [7]

$$
P=\frac{n 1}{n 2}
$$



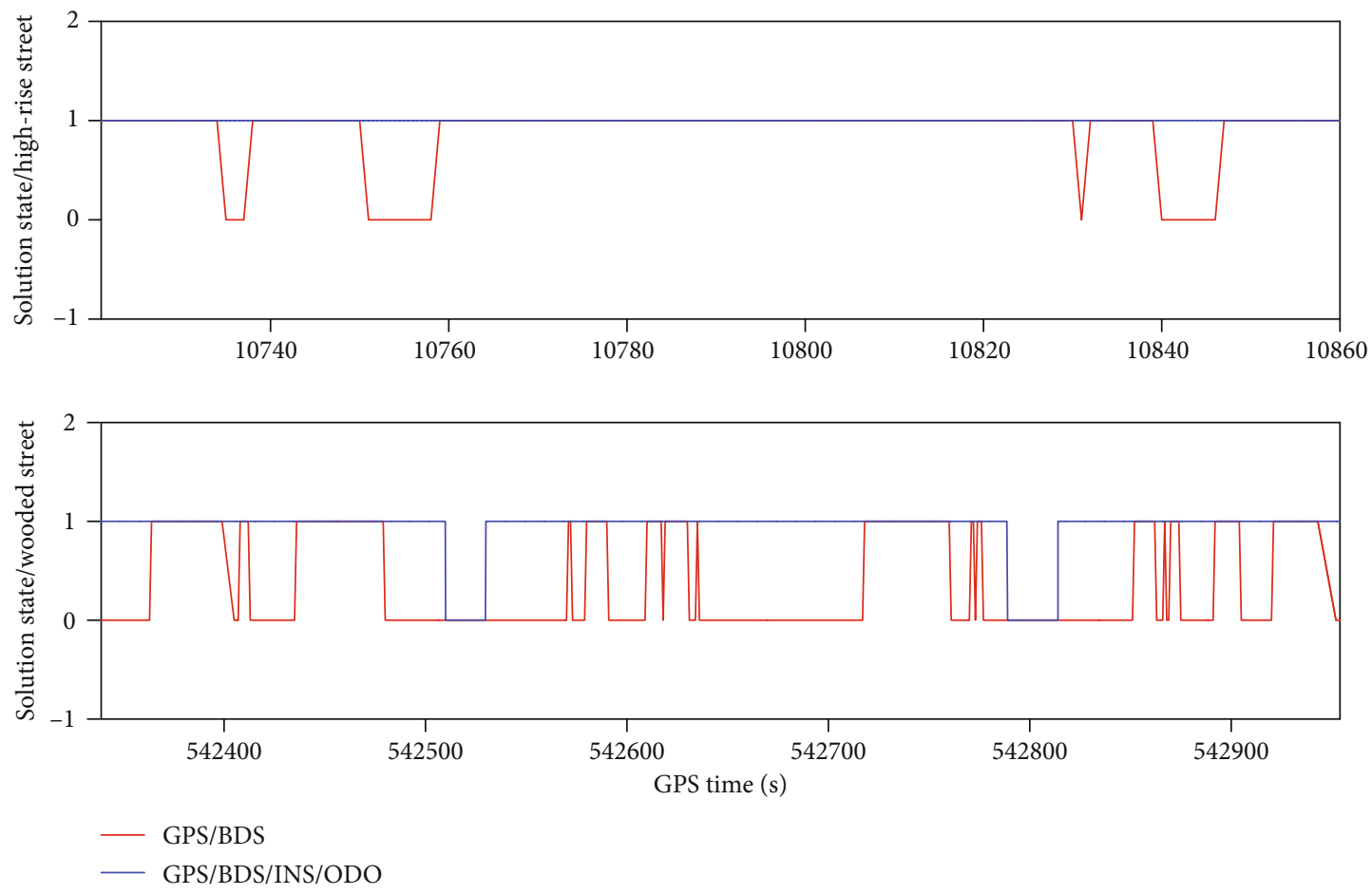

FIGURE 8: Solution state of AR for high-rise street and wooded street.

TABLE 5: AR empirical success rate of GPS/BDS/INS/Odometer in open environment, high-rise streets, wooded streets, and overpass in RTK mode.

\begin{tabular}{lcccc}
\hline Scene & $\begin{array}{c}\text { Open } \\
\text { environment }\end{array}$ & $\begin{array}{c}\text { High-rise } \\
\text { streets }\end{array}$ & $\begin{array}{c}\text { Wooded } \\
\text { streets }\end{array}$ & Overpass \\
\hline GC & $100 \%$ & $86.43 \%$ & $37.14 \%$ & - \\
GCIO & $100 \%$ & $100 \%$ & $92.08 \%$ & - \\
\hline
\end{tabular}

where $n 1$ is the number of the correctly fixed epochs and $n 2$ is the total number of epochs.

Table 5 shows the success rate for different scenes of open environment, high-rise streets, wooded streets, and overpass. GC represents GPS/BDS and GCIO for GPS/BDS/INS/Odometer. Because the satellite is in outage state under the overpass, the success rate is not evaluated here. In an open environment, the ambiguity fixing success rate for both models is $100 \%$. In the high-rise streets, the ambiguity fixing success rate of GCIO mode is $13.57 \%$ higher than that of GC mode. In the wooded street environment, the success rate has increased particularly significantly, by about 55 percent. Once again, it quantitatively shows that GCIO mode has great advantages in urban environment navigation.

3.3. Analysis of Positioning Performance. In this paper, the positioning and attitude accuracy of GC/GCIO combination in urban open environment, high-rise streets, wooded street, and urban tunnel are analysed. The details are as follows.
3.3.1. Urban Open Environment. In the open environment, the number of GPS/BDS satellites is large, the geometric structure is good, and the positioning accuracy is high. After the fusion of INS and odometer, the positioning accuracy is further improved, and the high-frequency attitude data is obtained, as shown in Figure 9.

The left column of Figure 9 shows the position deviation under the two combinations of GC/GCIO. From top to bottom, there are three axial positioning errors: the east, the north, and the earth. The attitude measurement deviation of the MEMS INS is shown in the right column. From top to bottom, there are three directions: heading, pitch, and roll. Table 6 shows the RMS (Root Mean Square) of the two combinations, the first three columns are the positioning RMS of the GC combination, and the last six columns are the pose measurement RMS of the GCIO combination. The latter scenarios are represented in the same way.

Figure 9 and Table 6 illustrate the vehicle pose measurement accuracy in the open environment. It can be seen that the RMS reaches the centimeter level in GC mode. Although the positioning accuracy is improved to a certain extent in GC and GCIO mode, the improvement effect is not obvious. In addition, the attitude accuracy is also very high, which proves the high accuracy of the algorithm again. This also shows that, if the vehicle is driving in the open environment, the positioning mode based on GPS/BDS/RTK mode can obtain higher absolute positioning accuracy.

3.3.2. High-Rise Street. Figure 10 and Table 7 show the positioning results of the two modes in high-rise street. 

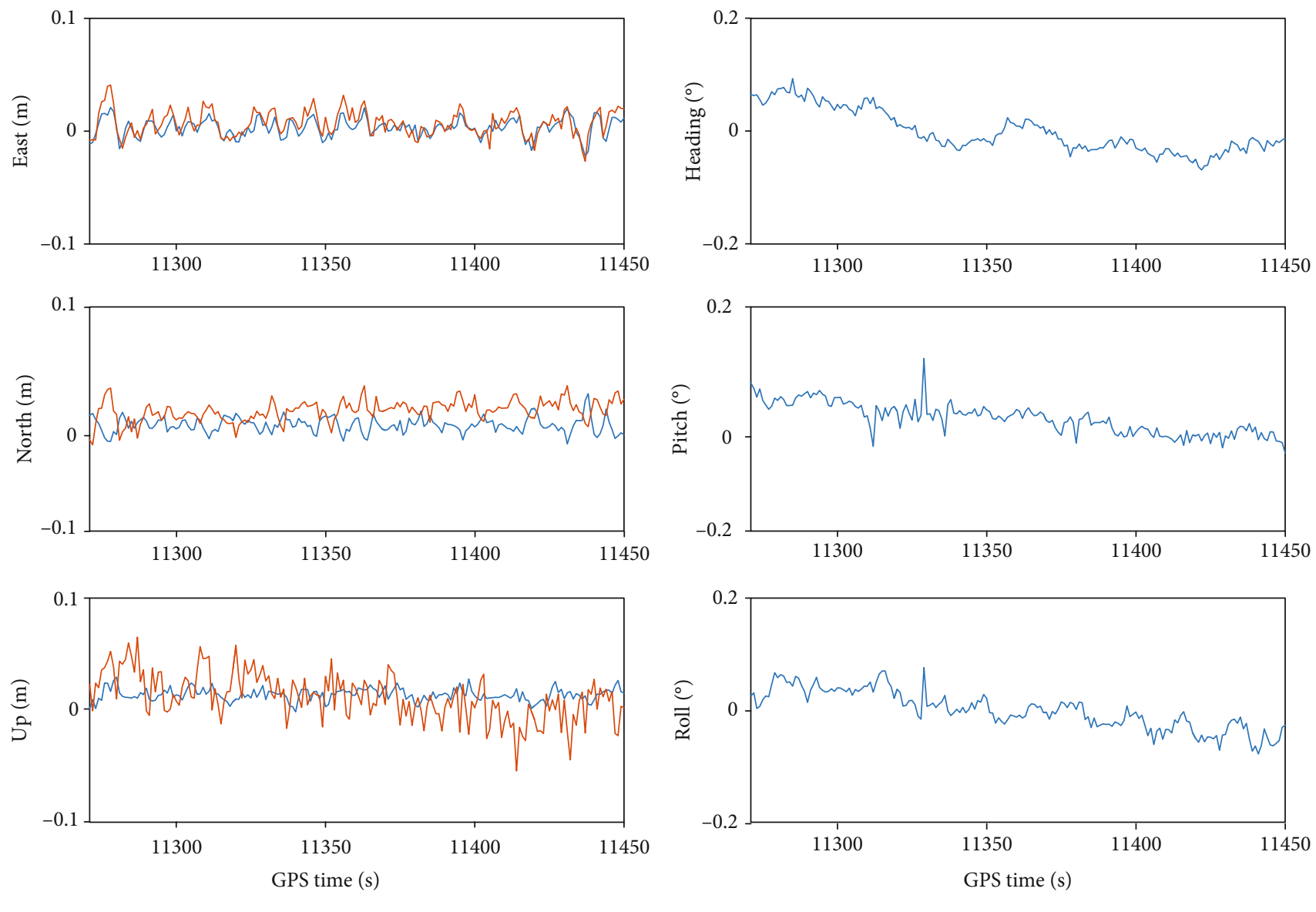

GPS/BDS/INS/ODO

- GPS/BDS/INS/ODO

FIgUre 9: Pose difference of GC/GCIO combination in urban open environment.

TABLE 6: RMS of the pose accuracy of GC/GCIO combination in urban open environment.

\begin{tabular}{cccccccccc}
\hline & & GC & & & \multicolumn{3}{c}{ GCIO } \\
& $\mathrm{E}(\mathrm{m})$ & $\mathrm{N}(\mathrm{m})$ & $\mathrm{U}(\mathrm{m})$ & $\mathrm{E}(\mathrm{m})$ & $\mathrm{N}(\mathrm{m})$ & $\mathrm{U}(\mathrm{m})$ & Heading $\left({ }^{\circ}\right)$ & Pitch $\left(^{\circ}\right)$ & Roll $\left(^{\circ}\right)$ \\
\hline RMS & 0.013 & 0.012 & 0.024 & 0.008 & 0.006 & 0.014 & 0.038 & 0.026 & 0.036 \\
\hline
\end{tabular}

Compared with the open environment, it can be seen that, affected by the buildings on both sides of the street, the positioning accuracy of GPS/BDS decreases obviously and the RMS in plane positioning is basically $0.3 \mathrm{~m}$, but the error in elevation is more than $1 \mathrm{~m}$, which is much larger than that in open environment. Based on the GCIO combination method, the positioning accuracy is significantly improved, the error amplitude becomes smaller, the RMS of plane positioning is increased by more than one order of magnitude, about $0.02 \mathrm{~m}$, and the elevation error is increased by $96.85 \%$. Compared with the open environment, although the accuracy is reduced by about twice, it can also basically meet the positioning requirements.

In addition, through the right column of Figure 10, it can be seen that the error in the horizontal attitude is about $0.35-0.5^{\circ}$ and the error in the heading angle atti- tude is about $0.074^{\circ}$. Moreover, the pitch and roll have always maintained a relatively stable state, and the heading deviation varies greatly. Compared with the open environment, it can be seen that the attitude accuracy of the vehicle is also greatly affected in the high-rise street environment, especially the heading angle, which is almost doubled.

3.3.3. Wooded Street. Due to the existence of a large number of green belts in the city, the streets are seriously blocked by the canopy, resulting in the blocking of satellite signals under the trees. Figure 11 and Table 8 show the pose measurement deviation under the combination of GC and GCIO of urban wooded street.

As can be seen from Figure 11, the positioning deviation is more serious based on GC combination under 

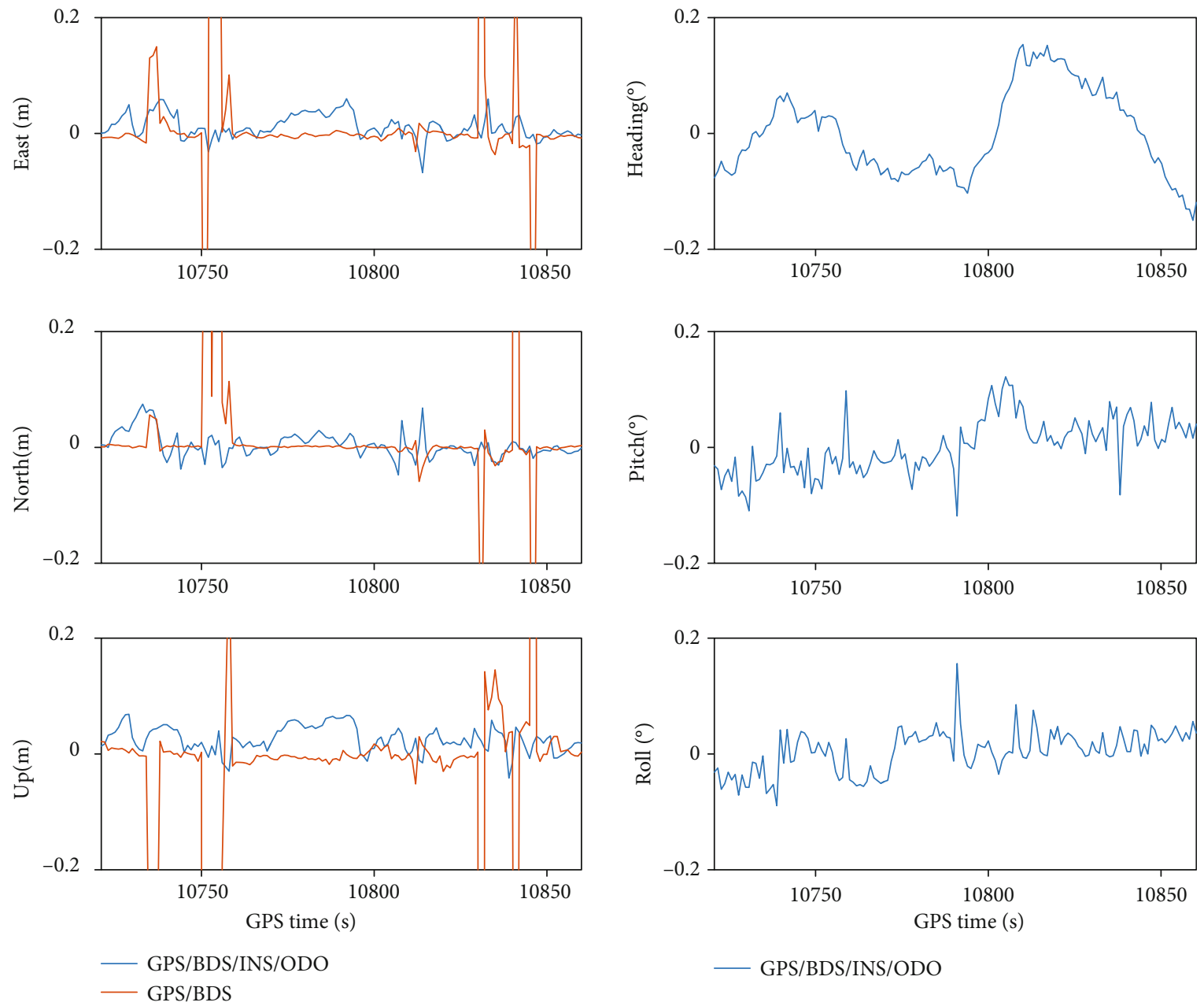

FIGURe 10: Pose difference of GC/GCIO combination in high-rise street.

TABLE 7: RMS of the positioning accuracy of GC/GCIO combination in high-rise street.

\begin{tabular}{cccccccccc}
\hline & & GC & & & \multicolumn{4}{c}{ GCIO } & \\
& $\mathrm{E}(\mathrm{m})$ & $\mathrm{N}(\mathrm{m})$ & $\mathrm{U}(\mathrm{m})$ & $\mathrm{E}(\mathrm{m})$ & $\mathrm{N}(\mathrm{m})$ & $\mathrm{U}(\mathrm{m})$ & Heading $\left({ }^{\circ}\right)$ & Pitch $\left(^{\circ}\right)$ & Roll $\left(^{\circ}\right)$ \\
\hline RMS & 0.256 & 0.361 & 1.047 & 0.023 & 0.018 & 0.033 & 0.074 & 0.050 & 0.035 \\
\hline
\end{tabular}

the wooded street. Combined with Table 8, it can be seen that the plane RMS is about 2 meters and the elevation RMS is 6.658 meters, indicating that dense trees have a serious impact on satellite positioning accuracy. On the other hand, the positioning accuracy of GCIO combined calculation is obviously improved to the order of $0.1 \mathrm{~m}$, which is increased by nearly 95\%, especially in the height direction by $98 \%$. But heading RMS is more than an order of magnitude lower than open environment and high-rise street.

3.3.4. Urban Overpass. Figure 12 and Table 9 show the pose measurement deviation under the urban overpass. The time period during which the GPS/BDS data is interrupted in Figure 12 is the time the vehicle is traveling under the overpass.
As can be seen from the figure, under the overpass, GPS/BDS is in the outage state and cannot produce effective positioning data. However, the positioning method based on GCIO combination can still achieve a positioning accuracy of $0.2 \mathrm{~m}$, which has a very good effect. This shows that under the environment of overpass, the combination method based on GCIO can solve the requirement of positioning. In addition, the attitude RMS is not only small but also stable, which further confirms the availability of GCIO. However, due to the short driving time under the overpass, the advantages of GCIO can only be explained to a certain extent. In view of the pose measurement deviation when the GNSS is in the outage state for a long time, we can refer to the urban tunnel environment.

3.3.5. Urban Tunnel. The tunnel test environment is Shanghai Dalian Road Tunnel, which is a north-south 

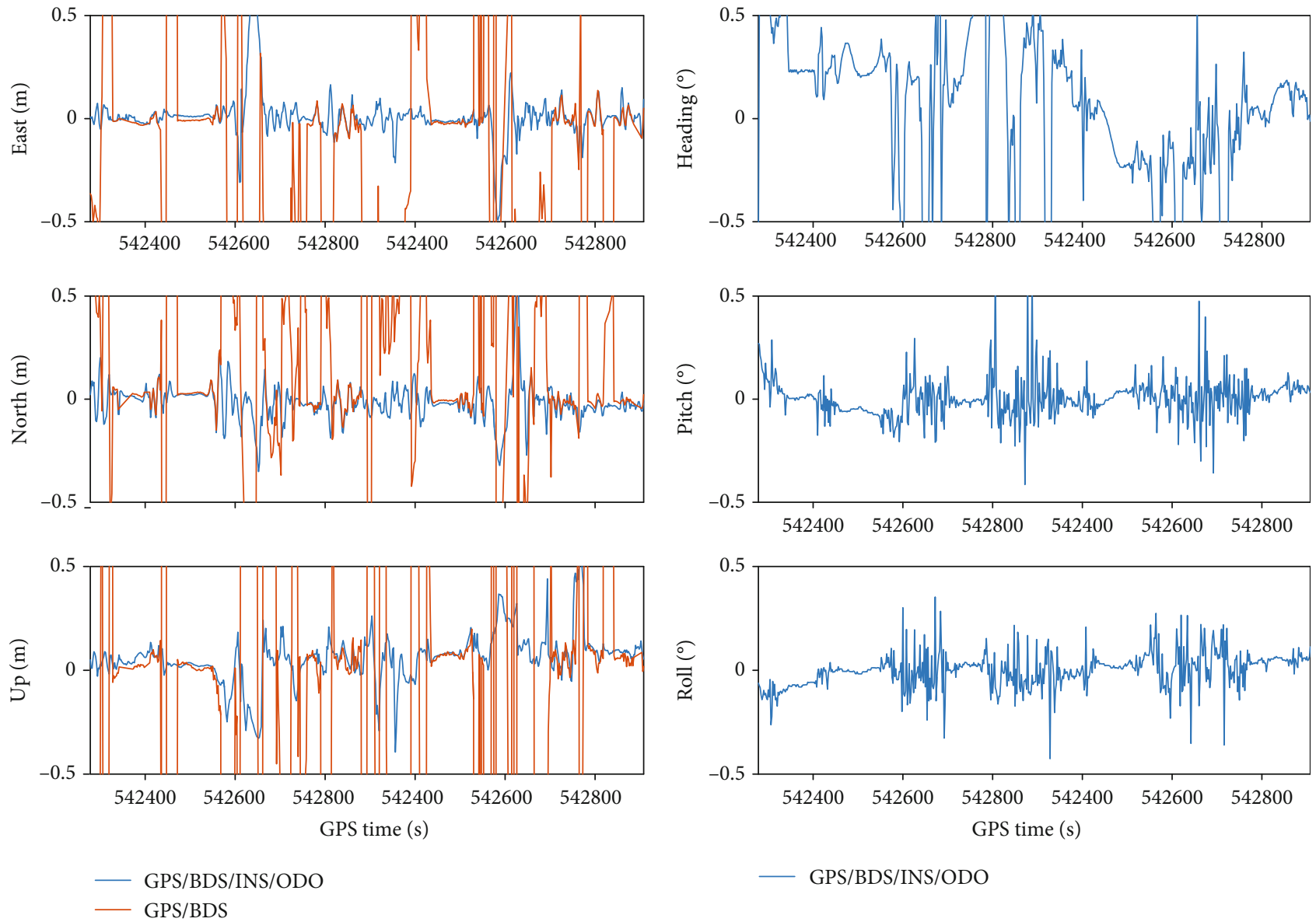

FIGURE 11: Positioning difference of GC/GCIO combination in wooded streets.

TABLE 8: RMS of the positioning accuracy of GC/GCIO combination in urban wooded street.

\begin{tabular}{cccccccccc}
\hline & & GC & & & & \multicolumn{3}{c}{ GCIO } & \\
& $\mathrm{E}(\mathrm{m})$ & $\mathrm{N}(\mathrm{m})$ & $\mathrm{U}(\mathrm{m})$ & $\mathrm{E}(\mathrm{m})$ & $\mathrm{N}(\mathrm{m})$ & $\mathrm{U}(\mathrm{m})$ & Heading $\left(^{\circ}\right)$ & Pitch $\left(^{\circ}\right)$ & Roll $\left(^{\circ}\right)$ \\
\hline RMS & 2.689 & 1.700 & 6.658 & 0.109 & 0.092 & 0.132 & 0.612 & 0.090 & 0.087 \\
\hline
\end{tabular}

tunnel located in Shanghai, China. It is the third underwater highway tunnel on the Huangpu River. Dalian Road Tunnel is $2526.88 \mathrm{~m}$ long and the design driving speed is $40 \mathrm{~km} / \mathrm{h}$. In the urban tunnel environment, GPS/BDS is in the state of outage, so it is impossible to compare the positioning accuracy of GC. Then, the pose measurement results of the self-developed module are compared with the FOG GNSS/INS combination equipment, and the pose measurement RMS is analysed when the GPS/BDS is in the state of outage for 10-120 seconds. The details are shown in Figure 13 and Table 10.

In the tunnel environment, we rely on INS and odometer for fusion positioning. It can be seen from Table 10 that the technical method of this paper can achieve submeter positioning accuracy in the range of 1 minute and better than 2 meters in 2 minutes. However, the deviation of RMS in the north direction is obviously higher than that in the east direction, which may be related to the accumulation of the error in the north direction when the vehicle is traveling from south to north. In addition, more than 1 minute later, the elevation accuracy has become poor and has exceeded 10 meters; there is still a lot of room for improvement. From the angle RMS, especially the heading angle, the error is reduced three times from 10 seconds to 2 minutes, but the RMS can still be maintained at $0.144^{\circ}$.

\section{Conclusions}

In order to solve the problem of high-precision navigation of vehicles in degraded and denied urban environment, a tightly coupled combination solution based on GPS/BDS/INS/Odometer is proposed in this paper. First of all, the combination of GPS/BDS is used to improve the number of satellites and the accuracy of DOP value in the occlusion environment of urban high-rise buildings. Secondly, the odometer is used to fuse with INS, the error of INS sensor in GPS/BDS outage environment is constrained, and the 

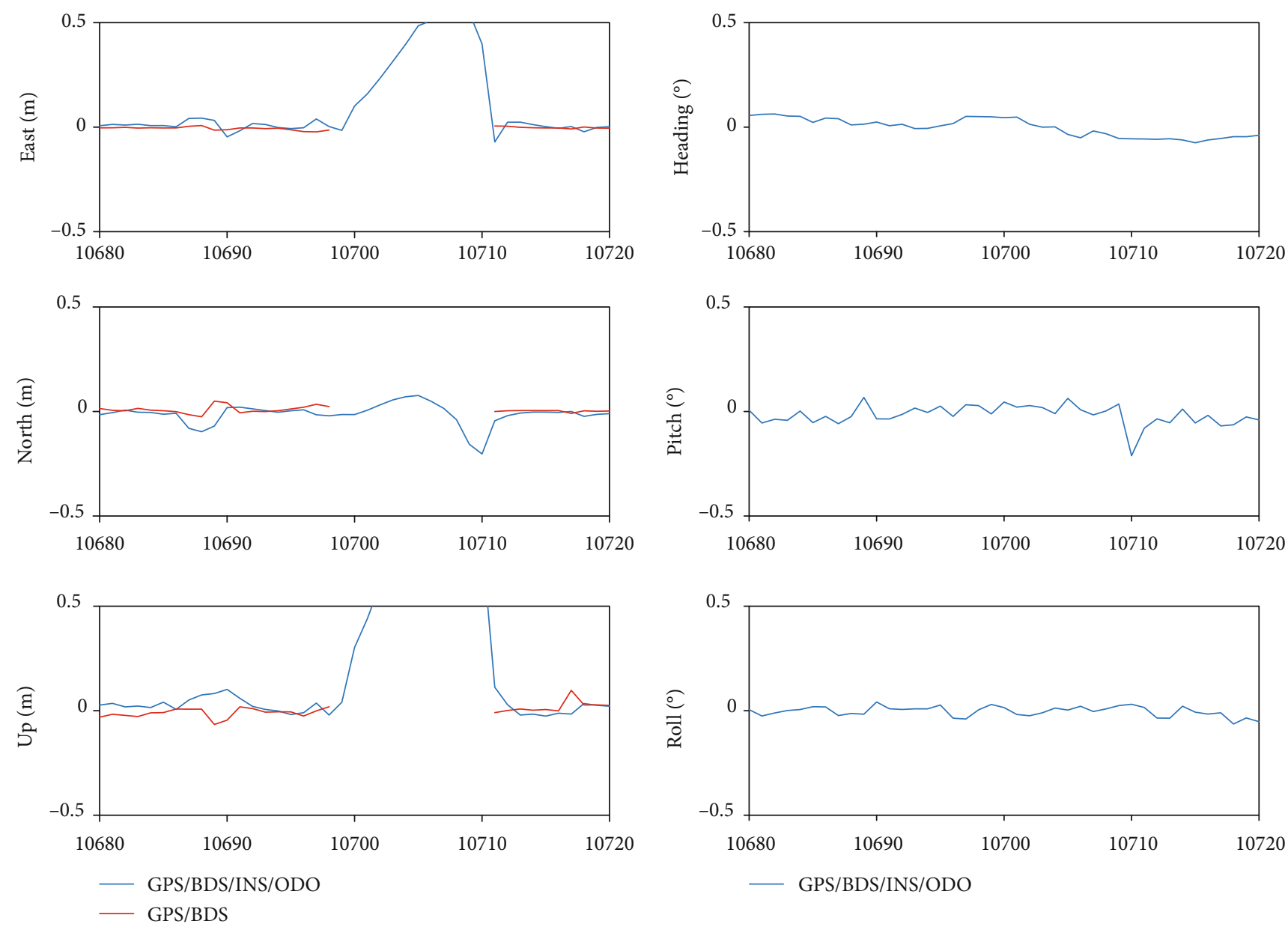

FIGURE 12: Positioning accuracy of GC/GCIO combination in urban overpass.

TABLE 9: RMS of the positioning accuracy of GC/GCIO combination in urban overpass.

\begin{tabular}{cccccccccc}
\hline & & GC & & & \multicolumn{4}{c}{ GCIO } & \\
& $\mathrm{E}(\mathrm{m})$ & $\mathrm{N}(\mathrm{m})$ & $\mathrm{U}(\mathrm{m})$ & $\mathrm{E}(\mathrm{m})$ & $\mathrm{N}(\mathrm{m})$ & $\mathrm{U}(\mathrm{m})$ & Heading $\left({ }^{\circ}\right)$ & Pitch $\left(^{\circ}\right)$ & Roll $\left({ }^{\circ}\right)$ \\
\hline RMS & - & - & - & 0.210 & 0.044 & 0.420 & 0.042 & 0.050 & 0.030 \\
\hline
\end{tabular}

fusion data is used to assist GPS/BDS AR, to ensure the fixed rate of ambiguity. Finally, the compact combination algorithm is used to realize multisensor fusion, making full use of the advantages of each sensor.

In addition, according to the experiment, we can sum up the conclusion as follows.

First of all, in the urban open environment, whether based on GPS/BDS or GPS/BDS/INS/Odometer positioning method, centimeter real-time positioning accuracy can be achieved, which can well meet the needs of vehicle navigation. Secondly, in the urban high-rise street, wooded street, overpass, and tunnel environment, the positioning accuracy of GPS/BDS will be affected; the most serious is the tunnel and wooded street; in the overpass because of the short driving time, the impact is low; the impact of high-rise street is the lowest, the positioning accuracy is close to that in the open environment. Finally, in the navigation mode based on GPS/BDS/INS/Odometer, no matter what kind of scene, the positioning accuracy has been greatly improved. In addition to the long tunnel environment within 2 minutes, which can maintain a positioning accuracy better than 2 meters, other scenes can basically achieve a positioning accuracy of 0.1 meters. However, because the experimental data in this paper are intercepted for a single scene, the number of samples is relatively small, so there is still a need for further experimental verification of a large number of data. Moreover, in the case of long-time GPS/BDS outage, there are still technical challenges in high-precision navigation for a vehicle. For this reason, it is necessary to introduce high-precision map, vision, laser auxiliary data, and sensors for further fusion, as well as vehicle motion models, such as 

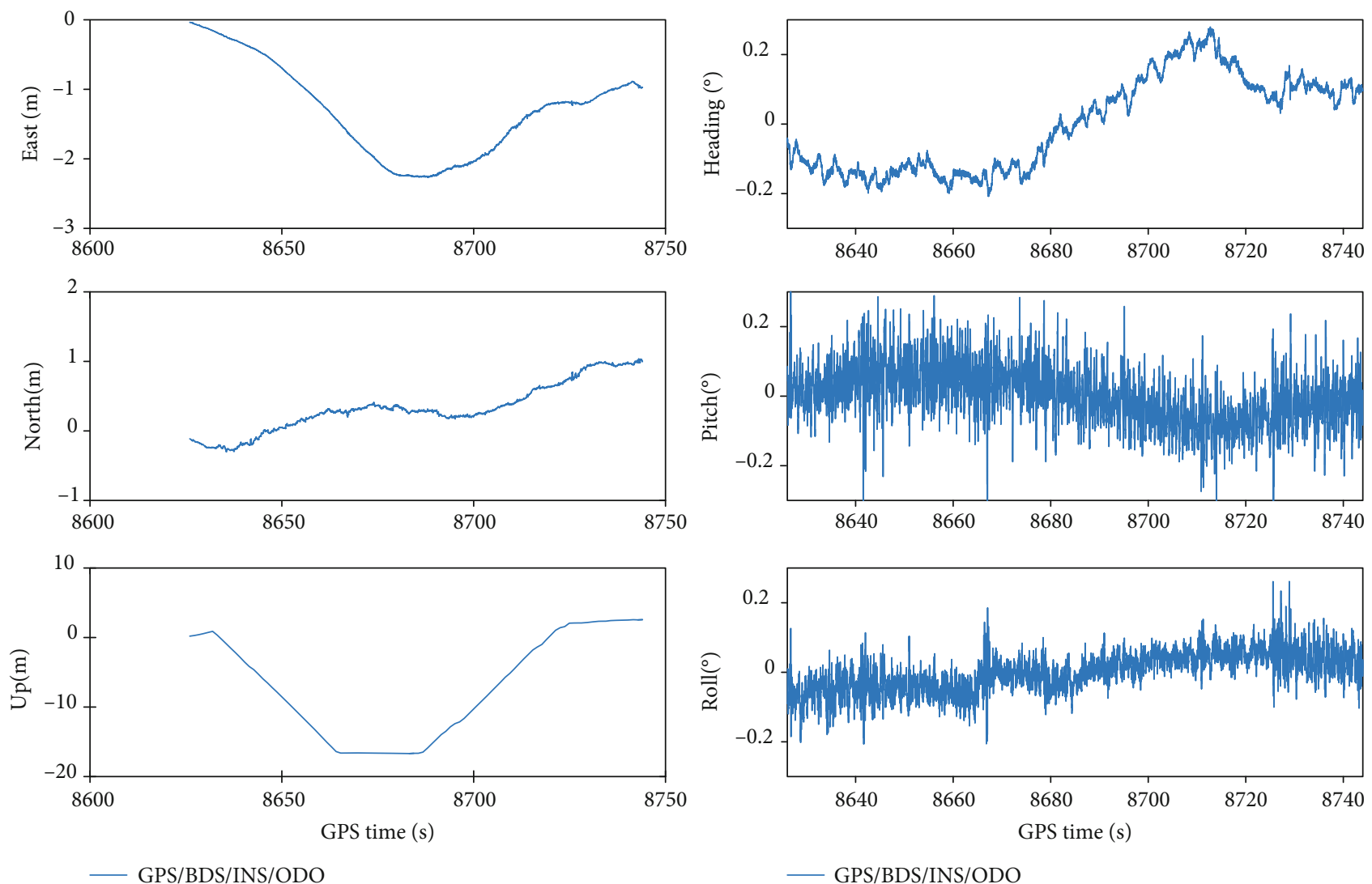

FIGURE 13: Pose difference of GCIO combination in urban tunnel.

TABLE 10: The pose measurement RMS of GCIO combination in urban tunnel.

\begin{tabular}{|c|c|c|c|c|c|c|}
\hline GC outage duration (s) & $\mathrm{E}(\mathrm{m})$ & $\mathrm{N}(\mathrm{m})$ & $\mathrm{U}(\mathrm{m})$ & Heading $\left({ }^{\circ}\right)$ & Pitch $\left({ }^{\circ}\right)$ & $\operatorname{Roll}\left(\left(^{\circ}\right)\right.$ \\
\hline 10 & 0.071 & 0.201 & 0.164 & 0.048 & 0.064 & 0.074 \\
\hline 30 & 0.203 & 0.308 & 1.398 & 0.134 & 0.066 & 0.067 \\
\hline 60 & 0.249 & 1.033 & 12.355 & 0.143 & 0.075 & 0.06 \\
\hline 120 & 0.328 & 1.735 & 12.386 & 0.144 & 0.081 & 0.057 \\
\hline
\end{tabular}

acceleration and deceleration and zero speed constraints, to further improve the positioning accuracy and retention in the case of GPS/BDS outage.

\section{Data Availability}

The data used to support the findings of this study are available from the corresponding author upon request.

\section{Conflicts of Interest}

The authors declare that there is no conflict of interest regarding the publication of this paper.

\section{Acknowledgments}

This research was funded by the National Science Foundation for Distinguished Young Scholars of China (grant number 41904029) and the National Natural Science Foundation of China (grant number 41874029).

\section{References}

[1] Q. Luo, Y. Cao, J. Liu, and A. Benslimane, "Localization and navigation in autonomous driving: threats and countermeasures," IEEE Wireless Communications, vol. 26, no. 4, pp. 3845, 2019.

[2] S. Campbell, N. O'Mahony, L. Krpalcova et al., "Sensor technology in autonomous vehicles: a review," in 2018 29th Irish Signals and Systems Conference (ISSC), pp. 1-4, Belfast, UK, June 2018.

[3] Y. Yang, J. Li, A. Wang et al., "Preliminary assessment of the navigation and positioning performance of BeiDou regional navigation satellite system," Science China Earth Sciences, vol. 57, no. 1, pp. 144-152, 2014.

[4] Y. Yang, Y. Xu, J. Li, and C. Yang, "Progress and performance evaluation of BeiDou global navigation satellite system: data analysis based on BDS-3 demonstration system," Science China Earth Sciences, vol. 61, no. 5, pp. 614-624, 2018.

[5] X. Li, X. Zhang, X. Ren, M. Fritsche, J. Wickert, and H. Schuh, "Precise positioning with current multi-constellation global 
navigation satellite systems: GPS, GLONASS, Galileo and BeiDou," Scientific Reports, vol. 5, no. 1, article 8328, 2015.

[6] R. Odolinski, P. J. G. Teunissen, and D. Odijk, "Combined BDS, Galileo, QZSS and GPS single-frequency RTK," GPS Solutions, vol. 19, no. 1, pp. 151-163, 2015.

[7] T. Li, H. Zhang, X. Niu, and Z. Gao, "Tightly-coupled integration of multi-GNSS single-frequency RTK and MEMS-IMU for enhanced positioning performance," Sensors, vol. 17, no. 11, p. 2462, 2017.

[8] X. Li, R. Jiang, X. Song, and B. Li, "A tightly coupled positioning solution for land vehicles in urban canyons," Journal of Sensors, vol. 2017, Article ID 5965716, 11 pages, 2017.

[9] H. Han, J. Wang, J. Wang, and X. Tan, "Performance analysis on carrier phase-based tightly-coupled GPS/BDS/INS integration in GNSS degraded and denied environments," Sensors, vol. 15, no. 4, pp. 8685-8711, 2015.

[10] C. Cristodaro, F. Dovis, G. Falco, and M. Pini, "GNSS receiver performance in urban environment: challenges and test approaches for automotive applications," in 2017 International Conference of Electrical and Electronic Technologies for Automotive, Torino, Italy, June 2017.

[11] L. Yang, Y. Li, Y. Wu, and C. Rizos, “An enhanced MEMSINS/GNSS integrated system with fault detection and exclusion capability for land vehicle navigation in urban areas," GPS Solutions, vol. 18, no. 4, pp. 593-603, 2014.

[12] Y. C. Tien, Y. L. Chen, and K. W. Chiang, "Adaptive strategybased tightly-coupled INS/GNSS integration system aided by odometer and barometer," ISPRS - International Archives of the Photogrammetry, Remote Sensing and Spatial Information Sciences, vol. XLII-2/W13, pp. 881-888, 2019.

[13] S. Godha and M. E. Cannon, "GPS/MEMS INS integrated system for navigation in urban areas," GPS Solutions, vol. 11, no. 3, pp. 193-203, 2007.

[14] A. Noureldin, T. B. Karamat, M. D. Eberts, and A. El-Shafie, "Performance enhancement of MEMS-based INS/GPS integration for low-cost navigation applications," IEEE Transactions on Vehicular Technology, vol. 58, no. 3, pp. 1077-1096, 2009.

[15] S. Chen, W. Jiang, B. Cai, J. Wang, and W. Shangguan, "Seamless train localization based on BDS/INS/odometer/MM multi-sensor navigation system," in Proceedings of International Global Navigation Satellite Systems, pp. 6-8, Colombo Theatres, Kensington Campus, UNSW Australia, December, 2016.

[16] Z. Li, J. Wang, B. Li, J. Gao, and X. Tan, "GPS/INS/odometer integrated system using fuzzy neural network for land vehicle navigation applications," Journal of Navigation, vol. 67, no. 6 , pp. 967-983, 2014.

[17] J. Park, D. Lee, and C. Park, "Implementation of vehicle navigation system using GNSS INS odometer and barometer," Journal of Positioning, Navigation, and Timing, vol. 4, no. 3, pp. 141-150, 2015.

[18] M. Aftatah, A. Lahrech, A. Abounada, and A. Soulhi, "GPS/INS/odometer data fusion for land vehicle localization in GPS denied environment," Modern Applied Science, vol. 11, no. 1, p. 62, 2016.

[19] N. Vagle, A. Broumandan, and G. Lachapelle, "Multi-antenna GNSS and inertial sensors/odometer coupling for robust vehicular navigation," IEEE Internet of Things Journal, vol. 5, no. 6 , pp. 4816-4828, 2018.
[20] A. Broumandan and G. Lachapelle, "Spoofing detection using GNSS/INS/Odometer coupling for vehicular navigation," Sensors, vol. 18, no. 5, p. 1305, 2018.

[21] N. Vavilova, V. Vyazmin, and A. Golovan, "Development of a low-cost INS/GNSS/odometer integration algorithm for a road surface testing laboratory software," in 2019 26th Saint Petersburg International Conference on Integrated Navigation Systems (ICINS), pp. 1-7, Saint Petersburg, Russia, May 2019.

[22] H. Han, J. Wang, J. Wang, and A. H. Moraleda, "Reliable partial ambiguity resolution for single-frequency GPS/BDS and INS integration," GPS Solutions, vol. 21, no. 1, pp. 251-264, 2017.

[23] A. El-Rabbany, Introduction to GPS: The Global Positioning System, Artech house, 2002.

[24] W. Li, W. Li, X. Cui, S. Zhao, and M. Lu, "A tightly coupled RTK/INS algorithm with ambiguity resolution in the position domain for ground vehicles in harsh urban environments," Sensors, vol. 18, no. 7, p. 2160, 2018.

[25] R. G. Brown and P. Y. C. Hwang, Introduction to Random Signals and Applied Kalman Filtering: With MATLAB Exercises and Solutions, John Wiley \& Sons, Inc., USA, 4th edition, 2002.

[26] B. Hofmann-Wellenhof, H. Lichtenegger, and J. Collins, "Global Positioning System," in Theory and Practice, Springer-Verlag Wien GmbH, 2001.

[27] H. Han and J. Wang, "Robust GPS/BDS/INS tightly coupled integration with atmospheric constraints for long-range kinematic positioning," Gps Solutions, vol. 21, no. 3, pp. 12851299, 2017.

[28] P. J. G. Teunissen, "An optimality property of the integer leastsquares estimator," Journal of Geodesy, vol. 73, no. 11, pp. 587593, 1999.

[29] G. Yan, Research on vehicle autonomous position and azimuth determining system, [Ph.D. thesis], Northwestern Polytechnical University, Xi'an, China, 2006.

[30] H. Han, Q. Wang, and J. Wang, "Robust ambiguity resolution for single-frequency GPS/BDS/MEMS IMU tightly coupled system," Journal of Chinese Inertial Technology, vol. 23, no. 4, pp. 493-499, 2015.

[31] I. Y. Bar-Itzhack and N. Berman, "Control theoretic approach to inertial navigation systems," Journal of Guidance Control Dynamics, vol. 10, pp. 1442-1453, 2012.

[32] D. A. Grejner-Brzezinska, R. Da, and C. Toth, "GPS error modeling and OTF ambiguity resolution for high-accuracy GPS/INS integrated system," Journal of Geodesy, vol. 72, no. 11 , pp. 626-638, 1998. 